\title{
Isolated Shoulder Palsy Due to Infarction of the Cortical Branch of the Anterior Cerebral Artery
}

\author{
Hiromasa Tsudaa, c, Takayuki Yamashita $^{\mathrm{a}}$, Hiroki Ogasawara ${ }^{\mathrm{b}}$
}

\begin{abstract}
An 85-year-old man with essential hypertension and diabetes mellitus abruptly developed right-sided isolated shoulder palsy (ISP). Cranial magnetic resonance imaging demonstrated infarction of the cortical branch of the left anterior cerebral artery (ACA), which involved the left precentral gyrus. In the primary motor cortex, the corresponding area to the shoulder is very small. Consequently, there have been only five reported cases of ISP due to a cortical infarction, and its vascular supply remains uncertain. Our present case denotes that the corresponding area to the shoulder in the primary motor cortex receives its blood from the cortical branch of the ACA.
\end{abstract}

Keywords: Homunculus; Magnetic resonance imaging; Precentral gyrus; Primary motor cortex; Pure motor monoparesis

\section{Introduction}

Isolated shoulder palsy (ISP) is defined as unilateral shoulder motor weakness without other neurologic deficit which is caused by pyramidal tract disorder [1-5]. In 1937, based on electrical stimulation of the brain surface during surgery, Penfield and Boldrey [6] reported that there was a broadly somatotopic representation of the different body parts in an arrangement in the primary motor cortex. The corresponding area to the shoulder is very small. Therefore, there have been only five reported cases of ISP due to a cortical infarction,

\footnotetext{
Manuscript accepted for publication December 9, 2013

${ }^{a}$ Department of Neurology, Tokyo Metropolitan Health and Medical Corporation Toshima Hospital, Japan

${ }^{\mathrm{b}}$ Department of Rehabilitation, Tokyo Metropolitan Health and Medical

Corporation Toshima Hospital, Japan

${ }^{\mathrm{c}}$ Corresponding author: Hiromasa Tsuda, Department of

Neurology, Tokyo Metropolitan Health and Medical Corporation

Toshima Hospital, 33-1, Sakaecho, Itabashi-ku, 173-0015 Tokyo,

Japan. Email: hiromasa_tsuda@tokyo-hmt.jp

doi: http://dx.doi.org/10.4021/jmc1634w
}

and its vascular supply remains controversial [1-5]. Here, we describe an additional case of ISP due to a cortical infarction, which proves that the corresponding area to the shoulder receives its blood from the cortical branch of the anterior cerebral artery (ACA).

\section{Case Report}

A 75-year-old Japanese man with essential hypertension and diabetes mellitus suddenly presented with difficulty in lifting the right arm, despite no shoulder pain in April 2013. Therefore, the patient was admitted to our neurologic ward. Consciousness was alert. Blood pressure was 144/84 mmHg. The heart rate was $62 / \mathrm{min}$. General examination demonstrated no abnormalities. Cranial nerve impairment was not detected. The patient was right-handed. Hand grasping power was 30 $\mathrm{kg}$ in the right and $26 \mathrm{~kg}$ in the left. In the upper extremities, right-sided Barre sign was positive. In the lower extremities, neither Barre sign nor Mingazzini sign was detected. Manual muscle testing on the right upper limb demonstrated that pectoralis major, deltoid, supraspinatus, infraspinatus, rhomboideus, serratus anterior and latissimus dorsi were at grade 4, despite trapezius, diceps brachii, triceps brachialis, brachioradialis, extensor carpi radialis longus, extensor carpi ulnaris, flexor carpi radialis and flexor carpi ulnaris at grade 5. Bend, extension and wiggle were normal in both sides of the fingers. Muscle tonus was normal in the extremities. Muscle atrophy and fasciculation were not observed. Deep tendon reflexes were all normal and pathologic reflexes were not detected in the extremities. Superficial sensation (touch sensation, pain sensation, temperature sensation and topesthesia), deep sensation (joint sensation and vibratory sense) and combined sensation (two-point discrimination, graphesthesia, stereognosis and double simultaneous stimulation) were all normal. Dysmetria, decomposition of movement and intension tremor were not observed in finger-nose test and nose-finger-nose test. Hyperpronation test and hand pronation supination test were all normal. Truncal ataxia was not observed. In the gait test, arm swing was slow on the right side. There were no other neurologic abnormalities. Based on these results, there were moderate weakness 


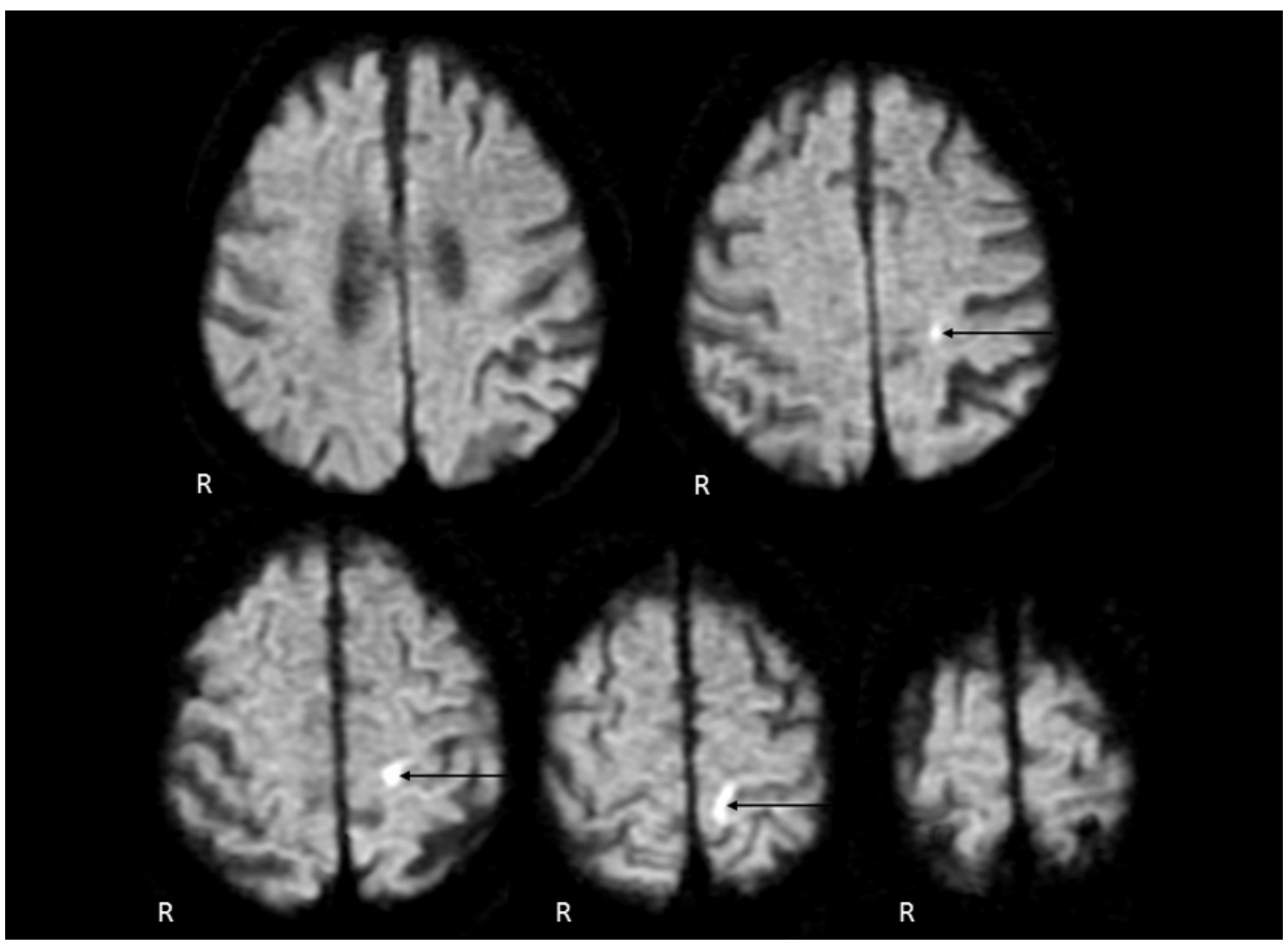

Figure 1. Cranial diffusion-weighted magnetic resonance imaging. An infarct lesion of the cortical branch of the anterior cerebral artery was demonstrated on the left side (arrow).

restricted to the right shoulder with normal joint flexibility, despite normal muscle strength in the elbow, wrist and fingers. Therefore, the patient was diagnosed as having rightsided ISP. Complete blood cell count was within normal ranges. Blood chemistry demonstrated elevated $\mathrm{HbA} 1 \mathrm{c}$ level at $6.4 \%$. Chest roentgenogram, electrocardiogram and echocardiogram findings were normal. Carotid ultrasonogram demonstrated a $3.9 \mathrm{~mm}$ diameter of hypoechoic plaque at the origin of the left internal carotid artery. Shoulder roentgenogram demonstrated no abnormalities. Cranial magnetic resonance (MR) imaging demonstrated an infarct lesion in the territory of the cortical branch of the ACA on the left side (Fig. 1). Cranial MR angiography demonstrated normal finding. By administration of intravenous ozagrel sodium 160 $\mathrm{mg} /$ day and the rehabilitation, marked blood pressure fluctuations were not observed and right-sided ISP was gradually resolved within 7 days. Thereafter, clopidogrel sulfate at $50 \mathrm{mg} /$ day was initiated for prevention of cerebral infarction.

\section{Discussion}

Penfield and Boldrey [6] described that the motor shoulder area might be located more medially than the motor hand area in the precentral gyrus. In addition, Celebisoy et al [7] noted that the hand area in the cerebral motor cortex was located in the middle to lower portion of the anterior wall of the central sulcus. However, in the cerebral motor cortex, detailed vascular supply remains uncertain. Kim [8] speculated that the medial portion of the precentral knob representing topographically ulnar-sided fingers may correspond to the borderzone area between the ACA and the middle cerebral artery (MCA), whereas the lateral portion representing radial-sided fingers is supplied by distal MCA branches.

In 2003, Komatsu et al [1] described a first case of ISP. Cranial MR imaging on the diffusion-weighted and T2-weighted demonstrated a small infarct lesion in the precentral gyrus on the ipsilateral side to shoulder palsy [1]. Echocardiogram did not demonstrate a source of embolus [1]. Borderzone infarction between the ACA and MCA was suspected as its pathogenesis, because of severe stenosis of the internal carotid artery based on MR angiography finding [1]. In 2006, Nah et al [2] noted a second case of ISP. Cranial MR imaging demonstrated a small infarct lesion in the right precentral gyrus [2]. Cranial MR angiography demonstrated no steno-occlusive lesions in the intracranial and extracranial arteries [2]. Electrocardiogram demonstrated atrial fibrillation [2]. Electrocardiogram demonstrated vigor- 
ous spontaneous echogenic contrast with sludge in the left atrial appendage [2]. However, detailed etiology of infarction was not mentioned [2]. In 2007, Uncini et al [9] reported a case of unilateral shoulder palsy due to a localized cortical infarction. An analysis of functional MR imaging confirmed the location of the corresponding area to the shoulder in the precentral gyrus [9]. However, muscle strength weakness of the biceps brachii and triceps brachii was also observed [9]. Pathogenesis of infarction was not described [9]. In 2011, Tsuda et al [3] noted a third case of ISP. Diffusion-weighted cranial MR imaging demonstrated a cortical infarct lesion in the two serial axial images [3]. Electrocardiogram, echocardiogram, carotid ultrasonography and cranial MR angiography demonstrated normal findings [3]. Marked blood pressure fluctuations were not observed. Consequently, atherothrombosis was suspected as pathogenesis of cortical infarction. However, whether the infarcted artery was originated from the ACA or MCA could not be specified, because the lesion was very small [3]. In 2012, Tsuda et al [4] reported a fourth case of ISP due to an infarction of the cortical branch of the MCA, which might be caused by atherothrombosis. In 2013, Tsuda et al [10] described a case of shoulder palsy as well as sensory aphasia due to MCA embolism. Therefore, Tsuda et al $[4,10]$ stated that the cortical branch of the MCA might supply blood for the corresponding area to the shoulder in the precentral gyrus. On the other hand, Kawasaki et al [5] noted a fifth case of ISP due to cerebral cortical infarction. Although cardiogenic embolism or Trousseau syndrome was suspected as its pathogenesis, infarcted artery could not be identified [5].

In our present patient with ISP, cranial MR imaging demonstrated an infarct lesion of the cortical branch of the ACA. Based on the carotid ultrasonogram finding, the infarction might be caused by artery to artery embolism. In conclusion, this is a sixth reported case of ISP due to a cortical infarction. We emphasize that the cortical branch of not only the MCA but also ACA might supply its blood for the corresponding area to the shoulder in the primary motor cortex.

\section{Conflict of Interest}

The authors state that they have no conflict of interest.

\section{Grant Support}

None.

\section{References}

1. Komatsu K, Fukutake T, Hattori T. Isolated shoulder paresis caused by a small cortical infarction. Neurology. 2003;61(10):1457.

2. Nah HU, Park HK, Kang DW. Isolated shoulder weakness due to a small cortical infarction. J Clin Neurol. 2006;2(3):209-211.

3. Tsuda H, Kubota Y, Tanaka K, Kishida S. Isolated shoulder palsy due to a cortical infarction. Intern Med. 2011;50(8):947.

4. Tsuda H, Shinozaki Y, Tanaka K, Miura Y, Kishida S, Karasawa K. Isolated shoulder palsy due to infarction of the cortical branch of the middle cerebral artery. Intern Med. 2012;51(16):2217-2219.

5. Kawasaki A, Suzuki K, Takekawa H, Kokubun N, Yamamoto M, Asakawa Y, Okamura M, et al. Isolated shoulder palsy due to cortical infarction: a case report and literature review of clinicoradiological correlations. J Stroke Cerebrovasc Dis. 2013;22(8):e687-690.

6. Penfield W, Boldrey E. Somatic motor and sensory representation in the cerebral cortex of man as studied by electrical stimulation. Brain. 1937;60(4):389-443.

7. Celebisoy M, Ozdemirkiran T, Tokucoglu F, Kaplangi $\mathrm{DN}$, Arici S. Isolated hand palsy due to cortical infarction: localization of the motor hand area. Neurologist. 2007;13(6):376-379.

8. Kim JS. Predominant involvement of a particular group of fingers due to small, cortical infarction. Neurology. 2001;56(12):1677-1682.

9. Uncini A, Caporale CM, Caulo M, Ferretti A, Tartaro A, Ranieri F, Di Lazzaro V. Isolated shoulder palsy due to cortical infarction: localisation and electrophysiological correlates of recovery. J Neurol Neurosurg Psychiatry. 2007;78(1):100-102.

10. Tsuda H, Nagao S, Yamazaki Y, Tanaka K. Shoulder palsy due to middle cerebral artery embolism. J Med Cases. 2013;4(2):86-88. 